\title{
Rapid measurement of the three-dimensional distribution of leaf orientation and the leaf angle probability density function using terrestrial LiDAR scanning
}

\author{
Brian N. Bailey ${ }^{\mathrm{a}, *}$, Walter F. Mahaffee ${ }^{\mathrm{a}}$ \\ ${ }^{a}$ USDA-ARS Horticultural Crops Research Laboratory, 3420 NW Orchard Avenue, \\ Corvallis, OR 97330, USA
}

\begin{abstract}
At the plant or stand level, leaf orientation is often highly anisotropic and heterogeneous, yet most analyses neglect such complexity. In many cases, this is due to the difficulty in measuring the spatial variation of the leaf angle distribution function. There is a critical need for a technique that can rapidly measure the leaf angle distribution function at any point in space and time. A new method was developed and tested that uses terrestrial LiDAR scanning data to rapidly measure the three-dimensional distribution of leaf orientation for an arbitrary volume of leaves. The method triangulates laser-leaf intersection points recorded by the LiDAR scan, which allows for easy calculation of normal vectors. As a byproduct, the triangulation also yields continuous surfaces that reconstruct individual leaves. In order to produce a probability density function for leaf orientation from triangle normal vectors, it is critical that the proper weighting be applied to each triangle. Otherwise, results will heavily bias toward normal vectors pointed toward the the LiDAR scanner. The method was validated using artificially generated LiDAR data where the exact

\footnotetext{
${ }^{*}$ Corresponding author

Email address: bnbailey@ucdavis.edu (Brian N. Bailey)

Current address: University of California, Davis, Department of Plant Sciences, Davis,
} CA 95616
\end{abstract}

Preprint submitted to Remote Sensing of Environment

September 9, 2016

(C) 2017. This manuscript version is made available under the Elsevier user license http://www.elsevier.com/open-access/userlicense/1.0/ 
leaf angle distributions were known, and in the field for an isolated tree and a grapevine canopy by comparing LiDAR-generated distribution functions to manual measurements. The artificial test cases demonstrated the consistency of the method, and quantitatively showed that errors in the predicted leaf angle distribution functions decreased as scan resolution was increased or as the density of leaves was increased. The isolated tree field validation showed qualitatively similar trends between manual and LiDAR measurements of distribution functions. Manual measurements of leaf orientation in the vineyard were shown to have large errors due to high leaf curvature, which illustrated the benefits of the more detailed LiDAR measurement method.

Keywords: Leaf angle distribution function, Leaf orientation measurement, Plant reconstruction, Terrestrial LiDAR scanning

\section{Introduction}

1 It is often necessary to account for the variability in leaf orientation in mete2 orological, climatological, or remote sensing analyses of plant systems. Because 3 the preferred orientation of leaves is driven by solar radiation, it is not surprising 4 that the probability density function (PDF) of leaf orientation is an important 5 component of radiation transport in plant canopies. Anisotropic leaf orientation generally results in anisotropic radiation transfer, which necessitates a descrip7 tion of leaf orientation in analysis (Ross, 1981; Myneni and Ross, 1991; Bailey 8 et al., 2014). Since leaf orientation dictates how light is reflected and scattered,

9 it is also an important consideration in the interpretation of remote sensing 10 data collected in vegetative environments (Nilson and Kuusk, 1989; Jones and 11 Vaughan, 2010). The preferred orientation of leaves also influences other pro12 cesses not related to radiation interception such as drag (Schuepp, 1993), particle deposition (Raupach et al., 2001), and convection (Schuepp, 1993; Bailey et al., 14 2016). 
Despite the known importance of leaf orientation in many environmental processes, previous work has been unable to faithfully represent the spatial and temporal variability of the leaf angle PDF in analyses. This is primarily due to the difficulty in obtaining adequate measurements of leaf orientation, which can be highly heterogeneous in space (Kull et al., 1999; Raabe et al., 2015) and variable over daily and seasonal scales (Shell et al., 1974; Thanisawanyangkura et al., 1997). In order to measure the leaf angle distribution function, a large number of individual measurements of leaf orientation can be collected to generate the PDF. Manual measurement of leaf orientation is extremely tedious, and can be difficult to perform in dense or tall vegetation. Manual measurements also have many potential sources for error, as each measurement requires a judgement call on the part of the individual. A passage from Norman and Campbell (1989) stresses these issues: "Direct measurement of leaf angle distributions requires much determination and extraordinary care; let the uninitiated beware."

The simplest and most common approach for dealing with such difficulties in analyses is to remove the effects of leaf orientation and make the assumption that leaves have no preferred orientation. This has been demonstrated to be a poor assumption in a vast number of cases (see Pisek et al., 2013). Regardless, it is an assumption that is often made out of necessity when measurements are unavailable. There is clear opportunity for improvement of many meteorological and climatological models, measurements, and general analyses by faithfully including the effects of leaf orientation.

In cases where some measurement of the leaf angle distribution function is desired but direct methods are not feasible, indirect methods have also been developed that use a collimated source of radiation at some known orientation to infer leaf angle distributions. The most easily accessible source of collimated 
radiation is sunlight. By recording how sunlight is attenuated by vegetation, one can invert the well known Beer's law for radiation interception to estimate leaf orientation (cf. Norman and Campbell, 1989). These methods are limited by the fact that all results are integrated along the path between the sun and the instrument, making it difficult to obtain three-dimensional information. They also generally require an estimation of the leaf area density (LAD), which can lead to compounding errors. A more recent indirect method that has gained popularity is to manually identify leaf angles from digital photographs (Ryu et al., 2010). This method is a bit more efficient than direct measurement, but it cannot measure leaf azimuth and only considers leaves in direct view that are approximately parallel with the camera view direction.

Terrestrial LiDAR scanning, which is the focus of this paper, has seen increasing usage in obtaining measurements of canopy structure (e.g., Radtke and Bolstad, 2001; Henning and Radtke, 2006; Hosoi and Omasa, 2006; Rosell et al., 2009; Yang et al., 2013). Terrestrial LiDAR scanners emit concentrated beams of radiation in the form of laser pulses into the spherical space surrounding the scanner. In the event that the beam hits an object, the distance to the object can be determined by analyzing the radiation reflected back to the scanner. Unlike methods utilizing the attenuation of sunlight, LiDAR can quickly obtain millions of data points that provide three-dimensional information about radiation attenuation from many directions, which can be inverted to provide information about canopy structure such as leaf area density (Hosoi and Omasa, 2006; Béland et al., 2011). One important limitation in the use of LiDAR data to measure LAD distributions is the general lack of knowledge of the behavior of the leaf angle distribution function, which plays a critical role in performing such measurements.

Some recent work has began developing methods to measure leaf orientation 
using LiDAR data. Zheng and Moskal (2012) estimated leaf normals using an algorithm that fits a plane to neighboring LiDAR leaf intersection points. The method performed well for small potted plants, but showed poor agreement with measurements when applied to a tree in the natural environment. Their method also did not properly weight leaf normals when calculating the PDF of inclination, leading to a biased PDF (see Sect. 2.2). Hosoi and Omasa (2015) manually identified leaves within LiDAR point cloud data, and used planar fits to calculate and average leaf normals. This method allows easy access to the entire plant, but it is comparable in effort to performing manual measurements in the field. As with any manual measurement technique, there is also the potential for user bias in results, which has not been quantified through validation exercises.

In this work, we seek to develop and validate an algorithm that autonomously calculates the three-dimensional distribution of leaf orientation in natural vegetation from LiDAR data. The ultimate goal is to rapidly generate the leaf angle PDF for an arbitrarily defined volume of leaves, providing a direct measurement of how the PDF changes in space and time.

\section{Methods}

\subsection{Triangulation and calculation of surface normals}

The LiDAR scan creates a uniform grid of sample points in spherical space. Let $\Theta$ and $\Phi$ denote the range of zenithal and azimuthal directions explored by the LiDAR, respectively. The number of scan points in the zenith $\left(N_{\Theta}\right)$ and azimuthal $\left(N_{\Phi}\right)$ directions gives the resolution of the scan. Thus, the zenithal angle between adjacent scan points is $\Delta \theta=\Theta / N_{\Theta}$, and the azimuthal angle is $\Delta \varphi=\Phi / N_{\Phi}$. For each of the $N_{\Theta} \times N_{\Phi}$ scan points, the instrument reports the distance to the nearest object in the direction $(\theta, \varphi)$. 
The strategy for computing the distribution of leaf normals was to first triangulate laser-leaf intersection points. A successfully constructed triangular mesh along leaf surfaces would allow for easy calculation of normal vectors at the location of each triangle. Triangles are desirable because they comprise the minimum number of points necessary to compute a normal vector. This means that only three adjacent hit points are required on a given leaf to calculate a normal vector and obtain an estimate of its orientation. Standard triangulation algorithms are not applicable for discrete surfaces such as leaves. Examples are (Press et al., 2007) Delaunay triangulation which in three dimensions seeks to create tetrahedral cells and not surfaces, or the convex hull algorithm which seeks to create a continuous convex surface. There are numerous algorithms designed to reconstruct continuous solid objects from point cloud data (e.g., Bernardini et al., 1999). Since plant leaves consist of a field of many individual surfaces, this work will develop a simpler algorithm below that is specifically designed to efficiently identify and reconstruct individual leaves without connecting each of them together to form a single object.

The scan data can be stored into a two dimensional data structure (denoted as $A_{i j}$ ), where columns denote each azimuthal scan direction and rows denote each zenithal scan direction (Fig. 1a and Table 1 ). If the $(i, j)$ scan point does not intersect an object (denoted as a 'miss'), $A_{i j}$ is set to false, whereas if the point intersects an object (denoted as a 'hit'), $A_{i j}$ is set to a unique identifier corresponding to the hit point. For practical purposes, a bounding box can be established that encompasses only points of interest, with all hit points lying outside of this box considered misses.

To triangulate the $(i, j)$ hit point, neighboring points in the table $A_{i j}$ were searched for hits (Fig. 1a and Table 1). If the $(i+1, j)$ and $(i+1, j+1)$ were both also hits, the $(i, j),(i+1, j)$, and $(i+1, j+1)$ points were connected 
(a)

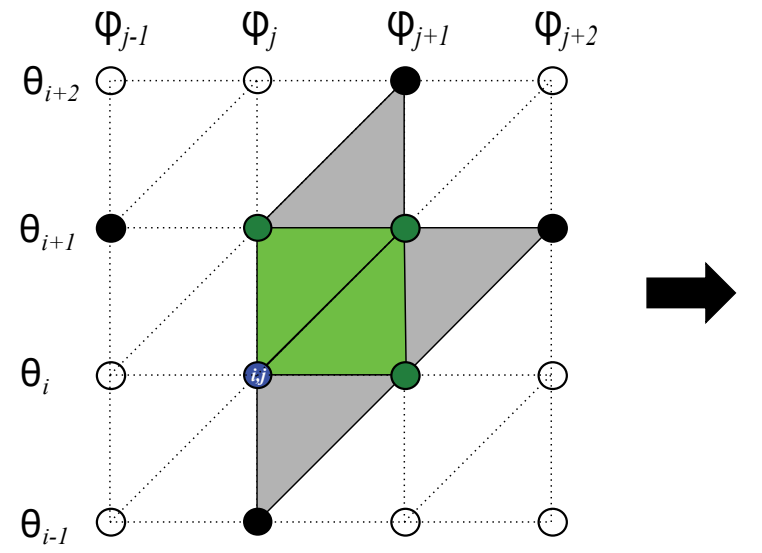

(b)

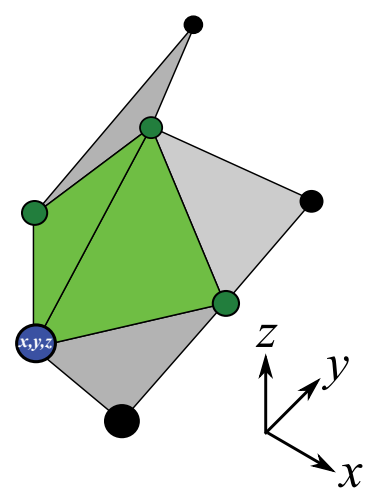

Figure 1: Visual depiction of point cloud triangulation. (a) shows how points are first triangulated using the two-dimensional grid of $\left(\theta_{i}, \varphi_{j}\right)$ pairs. Leaf-laser intersection points (hits) and non-intersection points (misses) are denoted respectively by closed and open circles. At any hit point, a triangulation is attempted with the $\left(\theta_{i}, \varphi_{j}\right),\left(\theta_{i+1}, \varphi_{j}\right),\left(\theta_{i+1}, \varphi_{j+1}\right)$ points and the $\left(\theta_{i}, \varphi_{j}\right),\left(\theta_{i+1}, \varphi_{j+1}\right),\left(\theta_{i}, \varphi_{j+1}\right)$ points. (b) shows the triangulation mapped to the $(x, y, z)$ hit coordinates.

to form a triangle. The $(i+1, j+1)$ and $(i, j+1)$ points were also searched, and if they were all hits, the $(i, j),(i+1, j+1)$, and $(i, j+1)$ points were connected to form another triangle. This proceeded for $i=1$ through $N_{\Theta}-1$ and $j=1$ through $N_{\Phi}-1$. A number of simple variations on this triangulation methodology are possible, such as attempting to form a triangle with the $(i, j)$, $(i+1, j)$, and $(i, j+1)$ points if the above triangles cannot be formed. This is usually only expected to add additional triangles on the edges of leaves, and overall we have found that using these slightly more complicated triangulation methods had a minimal impact on results.

Once triangles have been formed, their normal vectors are simply given by the cross product of the vectors defining any two sides of the triangle (Fig. 2)

$$
n=a \times b,
$$

${ }_{133}$ where $\boldsymbol{n}=\left(n_{x}, n_{y}, n_{z}\right)$ is the normal vector, and $\boldsymbol{a}$ and $\boldsymbol{b}$ are two vectors 
Table 1: Example table structure needed to perform the triangulation algorithm from LiDAR scan data depicted visually in Fig. 1. Mapping of hit indices in spherical grid space for all scan points. Numerical values correspond to point \# (row) in Table 2.

\begin{tabular}{l|cccc}
\hline \hline & $\varphi_{j-1}$ & $\varphi_{j}$ & $\varphi_{j+1}$ & $\varphi_{j+2}$ \\
\hline$\theta_{i+2}$ & 0 & 0 & 7 & 0 \\
$\theta_{i+1}$ & 1 & 4 & 6 & 8 \\
$\theta_{i}$ & 0 & 3 & 5 & 0 \\
$\theta_{i-1}$ & 0 & 2 & 0 & 0 \\
\hline \hline
\end{tabular}

Table 2: Example table structure needed to perform the triangulation algorithm from LiDAR scan data depicted visually in Fig. 1 . Storage of the $(x, y, z)$ position of each hit location (misses are not stored).

\begin{tabular}{c|ccc}
\hline \hline point \# & $\boldsymbol{x}$ & $\boldsymbol{y}$ & $\boldsymbol{z}$ \\
\hline 1 & $x_{1}$ & $y_{1}$ & $z_{1}$ \\
2 & $x_{2}$ & $y_{2}$ & $z_{2}$ \\
3 & $x_{3}$ & $y_{3}$ & $z_{3}$ \\
4 & $x_{4}$ & $y_{4}$ & $z_{4}$ \\
5 & $x_{5}$ & $y_{5}$ & $z_{5}$ \\
6 & $x_{6}$ & $y_{6}$ & $z_{6}$ \\
7 & $x_{7}$ & $y_{7}$ & $z_{7}$ \\
8 & $x_{8}$ & $y_{8}$ & $z_{8}$ \\
\hline \hline
\end{tabular}




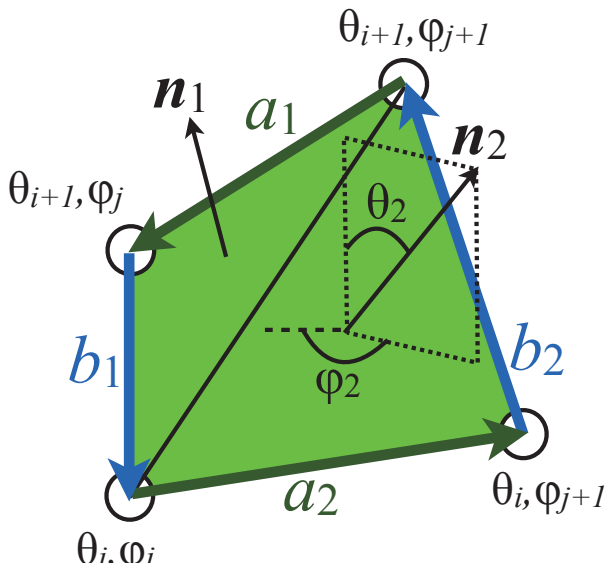

Figure 2: Schematic of the coordinate system for two adjacent triangles.

134

defining the sides of the triangle which are found by looking up the $(x, y, z)$ coordinates at each vertex (Table 2). The sign convention of the $\boldsymbol{a}$ and $\boldsymbol{b}$ vectors should be defined such that the calculated normal vectors are consistent (e.g., as in Fig. 2).

If desired, the triangle's zenithal and azimuthal angles can also be easily calculated from the normal vector as

$$
\theta_{L}=\operatorname{asin}\left(\frac{n_{z}}{|\boldsymbol{n}|}\right)
$$

and

$$
\varphi_{L}=\operatorname{atan}\left(\frac{n_{y}}{n_{x}}\right)
$$

In this work, we define $\varphi_{L}$ to range from 0 to $360^{\circ}$ such that it is consistent with the compass direction (0 is North, $90^{\circ}$ is East, etc.).

The LiDAR cannot directly determine if a hit point corresponds to the bottom or top surface of a leaf. In most applications, this is not problematic, as a distinction is often not necessary between the two sides of leaves in analyses. In 
order to approximately distinguish between the sides of a leaf, we assumed that all triangles with a downward pointing normal vector $\left(n_{z}<0\right)$ corresponded to the abaxial side of the leaf, and that $n_{z}>0$ corresponded to the adaxial side. If $n_{z}<0$, the normal vector was reversed (i.e., multiplied by -1), which effectively shifted the azimuthal angle by $180^{\circ}$. This allowed for direct comparison with manual measurements, which generally consider the leaf normal to be pointing away from the adaxial side of the leaf.

\subsubsection{Potential issues}

Leaf edge effects: It is clearly undesirable to have triangles that connect the edges of adjacent leaves, i.e., the triangles should stop at the edge of a leaf. Furthermore, the laser beam has a finite diameter (generally on the order of millimeters), which means that laser hits near the edge of the leaf will partially intersect the given leaf and partially intersect another object. Depending on the LiDAR instrument, it may effectively average multiple hit locations across the beam area and therefore partial hit points bias toward a location further along the beam path.

Although many possible methods could be devised to deal with the above complications, a relatively simple solution was used that is based on a size thresholding methodology. If any side of the triangle was longer than some predefined threshold length $L_{\max }$, the triangle was rejected. $L_{\max }$ should be defined such that it is significantly larger than the expected spacing between adjacent hit points on a leaf, yet significantly smaller than the expected spacing between adjacent leaves. As the scan density is increased, $L_{\max }$ becomes increasingly easier to define since the spacing between scan points decreases. It is certainly possible that this thresholding procedure will not eliminate some undesirable triangles such as when two leaves are very close to each other, however such instances should be relatively infrequent unless the canopy is extremely 
dense.

Noise in position measurements: The spatial locations of hit points have some error due to limited instrument accuracy, usually on the order of millimeters. Leaf motion due to wind also introduces some noise in the measurements. If the scan resolution is extremely fine, it is possible that the characteristic dimension of triangulated points can become near that of the spatial error in hit point measurements. If this occurs, calculated triangle normals become very sensitive to errors, and significant variation can be observed in the normals of two adjacent triangles on the same leaf. Some noise could potentially be reduced through smoothing (e.g., by weighted averaging adjacent normal vectors).

Woody material: In this work, no attempt was made to separate triangles associated with leaf and woody material. For plants with a large ratio of leaf to wood area, it may be acceptable to neglect the effects of woody material due to their negligible contributions. If the quantity of interest is simply net plant material, the distinction between the two may not be needed. If necessary, leaf and woody material could be separated using, e.g., the method of Béland et al. (2014).

Occluded leaves: An inherent limitation in any optical measurement is that objects occluded from the view of the sensor cannot be measured. This means that if there is significant heterogeneity in leaf orientation within the sampling volume, measurements are likely to bias towards leaves closest to the sensor. There are several means of reducing this bias. One is to use sufficiently small volumes that heterogeneity in leaf orientation is approximately resolved. This means that any sub-sample of a volume will be representative of the entire volume. Multiple scans can also be used to increase the number of samples in highly occluded volumes. Adding multiple scans can introduce the potential problem that some leaves within a volume may be sampled multiple times (oversampled). 
This can be considered analogous to the problem of under-sampling some areas of the volume, and means that a bias may be introduced if there is substantial heterogeneity in leaf orientation within a given volume.

\subsection{Formulating the probability density function}

A problem immediately arises when trying to use the normals of the triangulated points to form a PDF. As a leaf surface becomes increasingly inclined away from the scanner location, the distance between adjacent hit points on that leaf increases. The spacing between adjacent hit points is smallest when the leaf normal is parallel with the laser direction, and tends toward infinity as the leaf normal becomes orthogonal to the laser direction. In terms of the triangulation, this means that leaves with normals closer to parallel with the laser direction will have more triangles than leaves with normals closer to being orthogonal to the laser direction. As a result, the computed PDF will be biased toward leaves with normals that are parallel with the laser direction.

In order to correct for this bias, a triangle's normal should be weighted by its area. There are many ways to calculate the area of an arbitrary triangle, one example being Heron's formula

$$
A=\sqrt{s\left(s-\ell_{1}\right)\left(s-\ell_{2}\right)\left(s-\ell_{3}\right)},
$$

where $\ell_{1}, \ell_{2}$, and $\ell_{3}$ are the lengths of the sides of the triangle, and $s=$ $0.5\left(\ell_{1}+\ell_{2}+\ell_{3}\right)$.

The discrete leaf angle probability density function $g_{L}$ is then constructed by summing the total area of triangles within each discrete angle class, and normalizing such that $g_{L}$ integrates to unity. Regardless of the applied weighting, the method will still not account for the portion of leaves that are perfectly parallel to the laser direction, as these portions will have no hit points. 
In cases where there is a wide range of scan zenith angles for a given volume, an additional weighting may be needed to account for varying scan point density. Because of the scan pattern of the LiDAR, there is a higher density of scan points at lower zenith angles. Thus, triangles should also be weighted by $\sin \theta_{z}$, where $\theta_{z}$ is the zenith angle of the scan point.

\section{Validation}

\subsection{LiDAR instrument}

In this study, the Focus ${ }^{3 \mathrm{D}}$ X 330 (FARO Technologies, Lake Mary, FL, USA) portable laser scanner was used to map the structure of vegetation. The scanner emits laser pulses with a wavelength of $1550 \mathrm{~nm}$ in a uniformly gridded pattern over a field of view of $300^{\circ}$ in the vertical and $360^{\circ}$ in the horizontal (i.e., $\Theta=150^{\circ}$ and $\Phi=360^{\circ}$ ). The maximum scan resolution is $N_{\Theta} \times N_{\Phi}=17,080 \times$ 40,640 , which corresponds to an angular resolution of $\Delta \theta=\Delta \varphi=0.009^{\circ}$. The beam diameter at exit is roughly $2.25 \mathrm{~mm}$ and diverges at a typical half angle of about $0.011^{\circ}$. Upon intersection with an object (range from $0.6 \mathrm{~m}$ up to $330 \mathrm{~m}$ ), the three-dimensional coordinates of the intersection are calculated and recorded, with a typical distance accuracy of $\pm 2 \mathrm{~mm}$. The scanner also collects a large number of color digital photographs during the scan. This allows a mapping of the $3 \mathrm{D}$ hit point onto the $2 \mathrm{D}$ photographs, which gives an RGB color value associated with each hit point. The FARO unit records only one distance measurement per scan pulse. Any points deemed by the FARO firmware to correspond to multiple hits were automatically discarded, as they are assumed to be points on the edge of a leaf that will result in erroneous hit points as discussed previously in Sect. 2.1.1. More generally, if a LiDAR unit was used that could record multiple hits per pulse, the nearest hit location should be used in the triangulation. 


\subsection{Synthetic scan data}

Given the substantial errors expected for manual measurement of leaf orientation (e.g., Sect. 3.4), validation of the new method using field data presents a considerable challenge given that there is no 'gold standard' against which to compare. In order to address such problems, we formulated a novel methodology for validating the LiDAR scanning approach under ideal conditions. This involved creating synthetic LiDAR data that reproduces the scans that would be generated by the instrument described above. This methodology involved launching simulated rays from a given point and intersecting them with virtual leaves of known orientation. In this case, the virtual leaves were circular disks, which were chosen because simple ray-object intersection tests could be performed (cf. Suffern, 2007). Rays were launched in the same pattern as is done by the LiDAR instrument described above, which were intersected with each circular disk to determine the closest hit point (or that no hit occurred). The hit points were then triangulated, and the PDFs of the surface zenithal and azimuthal angles were computed.

A $1 \times 1 \times 1 \mathrm{~m}^{3}$ volume of disks was considered that was $3 \mathrm{~m}$ away from the simulated scanner and situated on the ground (Fig. 4a). The scanner location was $0.5 \mathrm{~m}$ above the ground. Disks with a radius of $5 \mathrm{~cm}$ were inserted into the volume with a uniform random position. The elevation and azimuthal angles of the disks were chosen by drawing from a uniform distribution. The performance of the triangulation method is dependent on the chosen leaf angle distribution, such that it will perform better for distributions that have more leaf area projected in the direction of the scanner. A uniform distribution was chosen so that equal weights are given to all orientations to avoid favoring certain orientations. Cases were simulated that had $2^{3}, 3^{3}, 4^{3}, 5^{3}$, and $6^{3}$ disks in the volume. Given the size of the volume $\left(1 \times 1 \times 1 \mathrm{~m}^{3}\right)$ and the area of the disks $\left(\pi(0.05)^{2} \mathrm{~m}^{2}\right)$, 
the one-sided area density of the cases were $0.063,0.021,0.50,0.98$, and 1.70 $\mathrm{m}^{2} / \mathrm{m}^{3}$. Since the random component means that results will change for each realization, 20 independent realizations were generated for each disk density.

\subsection{Field sites}

While the synthetic scan data can be used to demonstrate consistency of the method, there is still a need to perform field tests to ensure adequate performance in real vegetation. For this purpose, two validation experiments were performed. One was in a $6 \mathrm{~m}$ tall broad-leafed black cottonwood tree (Poplus balsamifera) in Oregon ( $\left.44^{\circ} 34^{\prime} 11.11^{\prime \prime} \mathrm{N}, 123^{\circ} 14^{\prime} 47.53^{\prime \prime} \mathrm{W}\right)$. The other was in a $2 \mathrm{~m}$ tall grapevine canopy (Vitis vinifera) near the isolated tree $\left(44^{\circ} 33^{\prime}\right.$ $\left.53.06 " \mathrm{~N}, 123^{\circ} 14^{\prime} 40.17^{\prime \prime} \mathrm{W}\right)$. The vines were trained to grow on a vertical shoot position (VSP) trellis system, with a row spacing of $2.5 \mathrm{~m}$. These two sites were chosen because they represented the range of possible leaf inclination classes, and were small enough to allow for the collection of direct measurements without the use of special equipment such as scaffolding. In both plants, the characteristic leaf dimension was on the order of $10 \mathrm{~cm}$, with some leaves as large as $15-20 \mathrm{~cm}$.

The tree was divided into three vertical levels, with four azimuthal zones at each level. The first vertical level began at $0.76 \mathrm{~m}$ from the ground to limit interference by grass and weeds, with each vertical level being $1.07 \mathrm{~m}$ tall. This left approximately $2 \mathrm{~m}$ of leaves above the top zone that were not measured since they could not be reached using the available equipment. The azimuthal zones faced each of the four cardinal directions. Manual measurements of inclination and azimuthal angle were performed on 50 randomly chosen leaves using a digital inclinometer and compass. The normal vector was taken to begin on the adaxial surface and emanate away from the leaf. Since all leaves had some degree of curvature, an 'average' leaf normal direction was estimated and measured. 
Four individual LiDAR scans of the tree were performed at each of the cardinal directions (which faced each zone), and co-registered together using the built-in GPS and inclinometer and refined by aligning common objects in the scene. The scanner was placed at roughly 2 meters from the nearest vegetation, and was mounted on a tripod about $1.5 \mathrm{~m}$ tall. Due to limited resources, only 6 of the 12 zones were sampled. These 6 zones were chosen in an alternating pattern: on the first level the North-South zones, second level the East-West zones, and third level North-South zones. Once leaf orientations had been measured, all leaves in each of the 6 zones were removed and scanned using an LI-3100C leaf area meter (LI-COR, Lincoln, NE, USA) to give a rough approximation of leaf area density, which was in the range of about $0.5-1.5 \mathrm{~m}^{2} / \mathrm{m}^{3}$ depending on the volume.

The vineyard was divided into 20 discrete volumes that were $0.76 \mathrm{~m}$ wide in the row-parallel direction. Each of these 20 volumes were divided into a lower and upper zone, resulting in 40 total zones. The lower zones started at the fruiting wire which was $0.76 \mathrm{~m}$ from the ground, and extended to a height of $1.17 \mathrm{~m}$. The upper zones started at a height of $1.17 \mathrm{~m}$ and extended to a height of $1.70 \mathrm{~m}$ which was the height of the uppermost catch wire. The top catch wire was chosen as a consistent boundary, although some vegetation extended above this height. In each of the 40 total zones, 10 manual measurements were made of leaf inclination and azimuthal angles using the same procedure as in the tree. The leaf orientation PDF was assumed to be homogeneous in the row-parallel direction, and all measurements at a given height were aggregated together. Two LiDAR scans were performed that were separated by $5 \mathrm{~m}$ in the row-parallel direction, which were co-registered using common objects in the scene. The scanner was placed in the middle of the row, which meant that it was roughly $1 \mathrm{~m}$ from the nearest vegetation. 
In the validation experiments, vegetation was first scanned with the LiDAR at three different densities: $N_{\Theta} \times N_{\Phi}=1708 \times 4064,2135 \times 5080$, and $3415 \times 8120$. For later reference, the resolutions of these scans will be respectively referred to as 'low', 'mid', and 'high'. The distance between adjacent scan points on a surface oriented normal to the scan direction can be roughly estimated as $\Delta s \approx r \Delta \theta$, where $r$ is the distance from the scanner. Note that strictly speaking this is only the distance between points in the $\theta$ direction, as the spacing in the $\varphi$ direction is a function of the scan zenithal direction. For the low and high resolution scans, $\Delta \theta$ is $1.6 \mathrm{mrad}$ and $0.82 \mathrm{mrad}$, respectively. This means that the closest leaf hit points for the tree (about $2 \mathrm{~m}$ away) have a spacing that ranges from about $3 \mathrm{~mm}$ to the width of the leaf $(\sim 10 \mathrm{~cm})$ depending on leaf orientation. For the furthest leaf hit points (about $6 \mathrm{~m}$ away), the hit point spacing ranges from about $1 \mathrm{~cm}$ to the width of the leaf. When the high resolution scan was used, these values are reduced by about a factor of 2 . Based on these estimations, $L_{\max }$ was chosen to be $5 \mathrm{~cm}$. For this value, erroneous triangles connecting leaves spaced at larger than $5 \mathrm{~cm}$ will be eliminated. This does mean that there may be some triangles on leaves within a few degrees parallel with the scan direction that will be undesirably eliminated.

The scan rate was set to $122 \mathrm{~K}$ points/second, which, considering the scan pattern, means that it takes around 0.05 sec to scan two adjacent azimuthal directions, thus this is the time to scan the four adjacent points needed to construct a triangle. As a result, wind may become a factor if leaf movement is substantial over this period of time. The leaf should not move faster than a few $\mathrm{cm} / \mathrm{s}$, otherwise the distance moved could be comparable to the triangle dimension. 


\subsection{Estimation of errors in manual measurements}

Manual measurements of leaf orientation contain substantial errors that can vary between the individuals that are performing the measurements. The foremost of which is that each measurement requires a judgement call by the individual. Any leaf has an infinite number of normals, and the measurer must assign a single value (or some finite number of values). For leaves with minimal curvature, this is not particularly problematic as the range of possible normals on a single leaf is small. However, for leaves with substantial curvature, it is not uncommon to find leaves with inclination angles that span the full range of 0 to $90^{\circ}$. Thus, an individual's perception of the 'average' normal is quite subjective.

For the isolated tree, leaves had minimal curvature and thus choosing a representative normal vector was relatively consistent. However, the vineyard leaves were highly complex, often resulting in ambiguous normal vectors. To obtain some estimation of the human error in manual measurements in the vineyard canopy, several raters were asked to measure the inclination and azimuthal angles of the same 20 leaves. Each rater used the same equipment, and were given the same set of instructions. They were told to imagine an 'average' plane passing through the leaf, whose normal vector they were to measure. They were instructed to resolve any ambiguity using their best judgement.

The inter comparison of measurements shows a considerable amount of variance in some cases (Fig. 3). The standard deviation between raters for all leaves was $7.3^{\circ}$ and $14.7^{\circ}$ for leaf inclination and azimuth, respectively. If these values are normalized by the range of possible values, the normalized standard deviations are found to be about $8 \%$ and $4 \%$ for leaf elevation and azimuth, respectively.

Certain leaves had inclinations that appeared to be less ambiguous to in- 

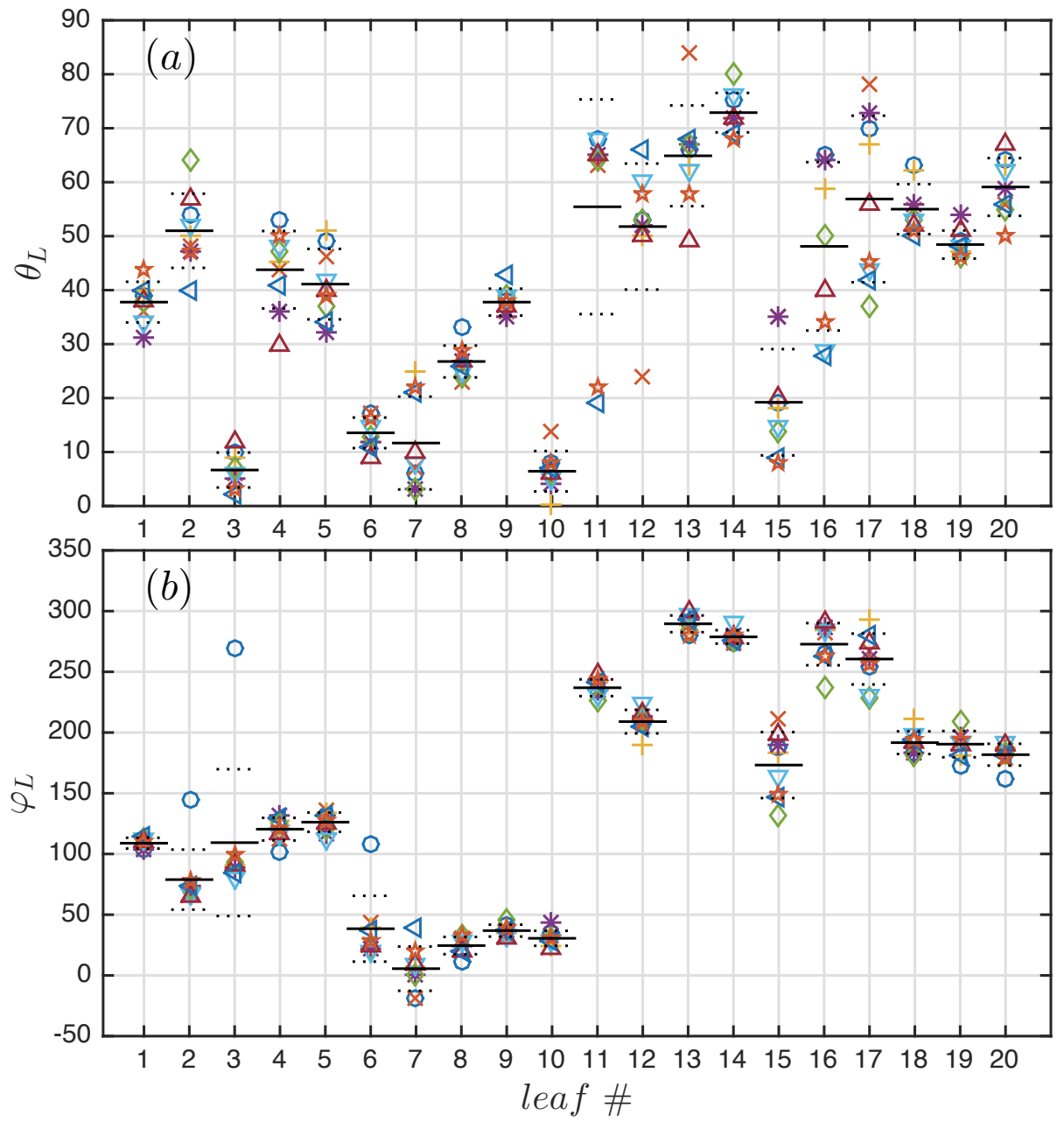

Figure 3: Assessment of error in manual measurements of leaf orientation in the vineyard canopy. Differing symbols correspond to each of the nine raters, who measured the same 20 leaves in the vineyard. Measurements are reported of the person's view of the average or representative (a) leaf inclination and (b) leaf azimuth. Solid horizontal lines denote the mean observation, and dashed horizontal lines denote \pm 1 standard deviation. Note that some azimuthal measurements in (b) were shifted by $360^{\circ}$ such that they could be visually grouped with other measurements. 
dividual raters than others, and had a standard deviation of only a couple of degrees (e.g., leaves 6, 9, 19). The elevation of other leaves appeared to be quite ambiguous. For example, the sampled distributions for leaves 16 and 17 appeared to be bi-modal. For leaf 17 about half of the group found that the leaf was inclined at about $75^{\circ}$, while the other half found that the leaf was inclined at about $40^{\circ}$. This result can likely be explained by the curvature of the leaves. By examining the structure of grapevine leaves, we found that a 'characteristic' leaf had the two lobes nearest the petiole oriented close to vertical, the mid portion of the leaf inclined at around $45^{\circ}$, and the lobe furthest from the petiole inclined somewhere between $45-90^{\circ}$. There are certainly many exceptions to this characterization, but this appeared to be the most representative view.

Considering the measurement of the 'characteristic' leaf, there are several likely scenarios. First is that a rater could choose to measure the center of the leaf, in which case they will likely find an inclination angle nearer to $45^{\circ}$. They could also choose the more vertical portion of the leaf, in which case they will likely find an inclination angle nearer to $90^{\circ}$. If the rater was to take a true average of the leaf inclination, they would find an average inclination angle of somewhere between $45-90^{\circ}$, most likely around $70^{\circ}$.

It appears to be quite difficult to assign an 'average' normal to a leaf with substantial curvature. For the grapevine canopy, manual measurements are likely to bias toward the center of the distribution. This underscores the need for a measurement method that eliminates non-systematic bias. Some additional accuracy could likely be gained by making multiple measurements on a single leaf, but this significantly increases the time required to perform measurements, and it still does not eliminate non-systematic bias. 


\section{Results}

\subsection{Synthetic scans}

Synthetic scan data was generated with densities that matched the 'low' and 'high' resolution scans used in the field experiments. For consistency with the field experiments, $L_{\max }$ was also chosen to be $5 \mathrm{~cm}$. Sample PDFs are shown in Fig. 4 for 2 leaves (Fig. 4b,c) and 27 leaves (Fig. 4d,e). In the 2 leaves case, disk directions were not randomly chosen, but rather were chosen such that one disk had most of its area projected normal to the scanner $\left((\theta, \varphi)=\left(80^{\circ}, 0\right)\right)$, while the other disk had little area projected normal to the scanner $((\theta, \varphi)=$ $\left.\left(10^{\circ}, 170\right)\right)$.

Results are compared for cases when equal weights are incorrectly applied to all triangles, and when the PDF is correctly area weighted. As expected, the equally weighted PDF biases toward triangles that are closer to being orthogonal to the ray direction. We incorrectly observe that it is almost three times more likely to find a triangle inclined at $10^{\circ}$ versus $45^{\circ}$. When the proper area weighting is applied, we obtain a computed PDF that is very close to the exact PDF. Small errors are due to the slightly jagged nature of the triangulation around the edges of the disks, and due to would-be triangles that are obstructed from the view of the scanner.

The effects of obstructed disks was tested by varying the density of disks within the volume (Fig. 5). As the density of disks within the volume increases, the average error in the predicted PDF decreases. For a small number of disks (e.g., 8), 'luck' has a considerable effect on the accuracy of the predicted PDF. If, by chance, most of the disk area is unobstructed from view of the scanner and there are no disks nearly parallel to the scan direction, errors were quite low. A significant number of obstructed or nearly parallel disks could also cause errors to be very large. As disk density was further increased, results became 
more consistent. In these cases a substantial fraction of disk area is consistently obstructed, but there are enough samples in view to obtain a good approximation of the PDF. In examining several of the individual PDFs, we found that the first $\theta_{L}$ bin was consistently under predicted. This bin corresponds to leaves inclined nearly parallel to the scan direction. It should be noted, however, that depending on the position of the leaves relative to the scanner, the first $\theta_{L}$ bin may not always correspond to leaves nearly parallel to the scan direction.

\subsection{Isolated tree}

A visualization of individual hit points colored by their RGB color value is shown in Fig. 6a. The visualizations revealed that leaves located in the outerlower portion of the tree tended to be oriented closer to vertical. Qualitatively, leaves in the upper portion of the tree were generally more horizontal. Average leaf azimuth tended to point along the shortest path length leading to the outer edge of the crown.

Calculated leaf angle PDFs provided a more quantitative depiction of trends in leaf orientation (Figs. 9 and 10). The average leaf inclination angle in the lower portion of the tree was greater than $45^{\circ}$, and was close to 'erectophile' as classified by de Wit (1965) (Fig. 8a). Leaf azimuthal angles tended toward the outward-facing direction of the tree crown (Fig. 8b). Moving upward along the tree, the leaf inclination PDF becomes skewed toward the horizontal orientation (planophile). Furthermore, the PDF of leaf azimuth becomes closer to uniform.

In the upper crown, leaves in the East- and West-facing zones tended to have azimuthal distributions that skewed toward the South.

These trends are intuitively consistent with configurations that are likely to maximize solar interception. For an unobstructed solar collector at the geographical location of the tree, the optimal normal direction is nearly Southfacing with an inclination of about $35^{\circ}$ (Lave and Kleissl, 2011). Leaves in the 

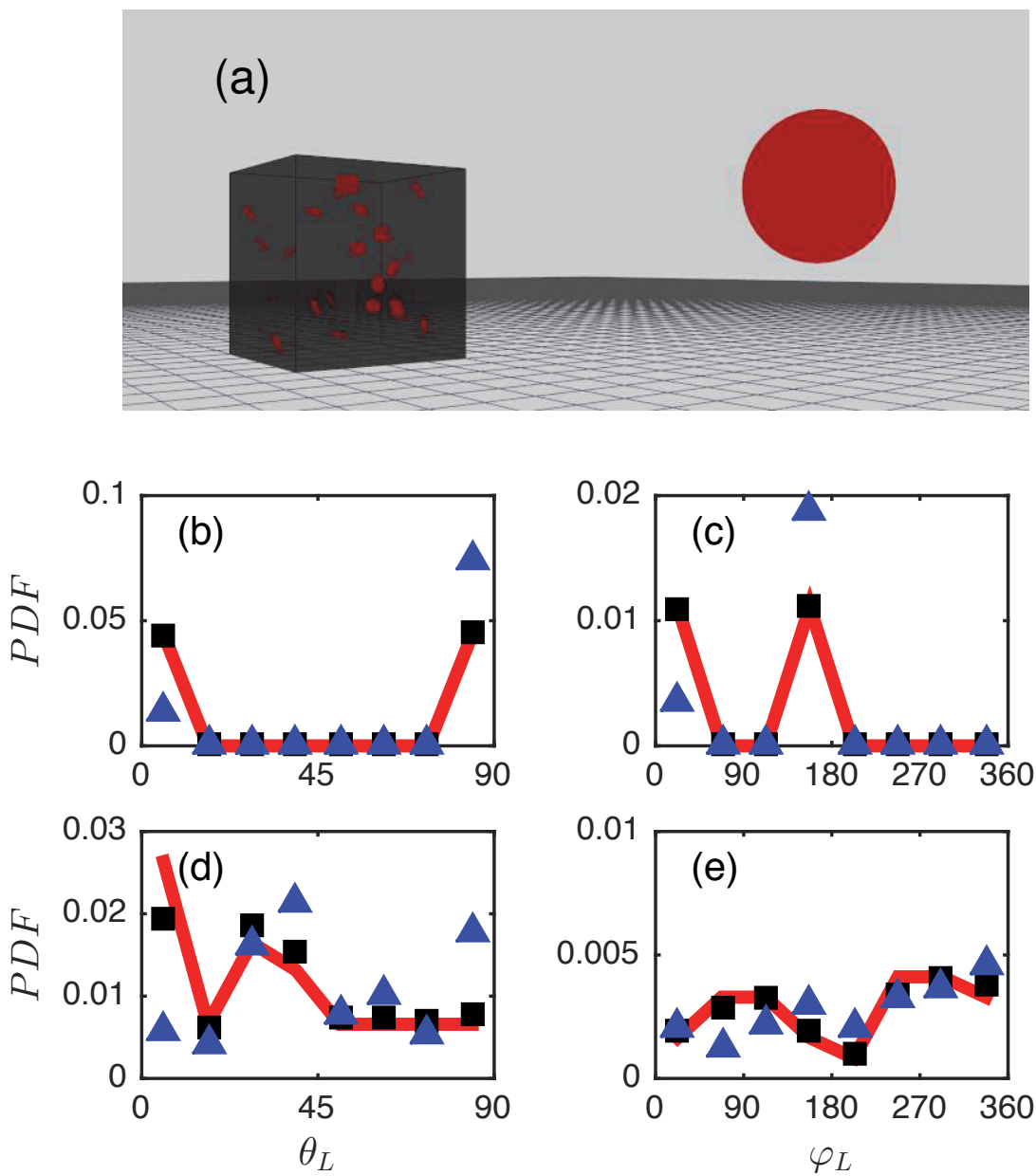

exact

area weighted

unweighted

Figure 4: Illustration of triangulation methodology using synthetic LiDAR scan data. A visual depiction of the simulated scan with 64 circular disks is shown in (a). Rays are launched from the sphere, some of which intersect the disks, which are visualized using the triangulation. Probability density functions are calculated when no weighting is applied and when a proper area weighting is applied, which are compared to the 'exact' distributions. (b,c) 2 disks, and (d,e) 27 disks. 

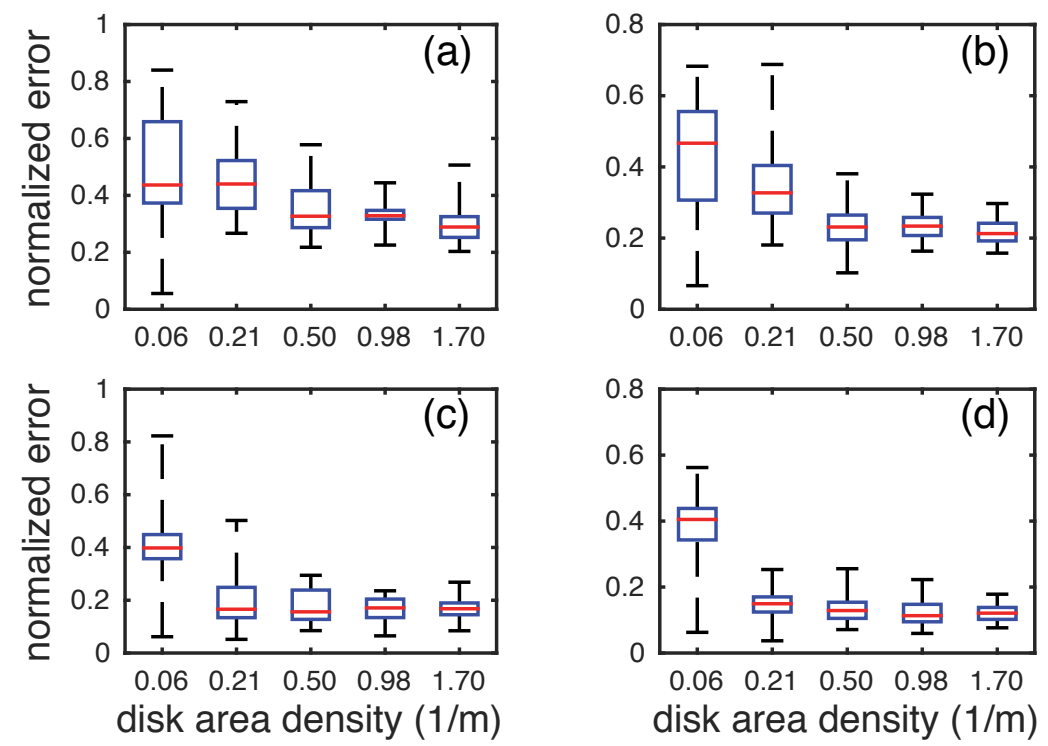

Figure 5: Box-and-whisker plots of normalized error in the PDF of (a,c) $\theta_{L}$ and (b,d) $\varphi_{L}$ for the synthetic scan cases. The normalized error is defined as the root-mean-squared error between the exact PDF and the PDF formed by the triangulation methodology, which is normalized by the probability density for a uniform distribution (i.e., $1 / 90$ for $\theta_{L}$ and $1 / 360$ for $\left.\varphi_{L}\right)$. 

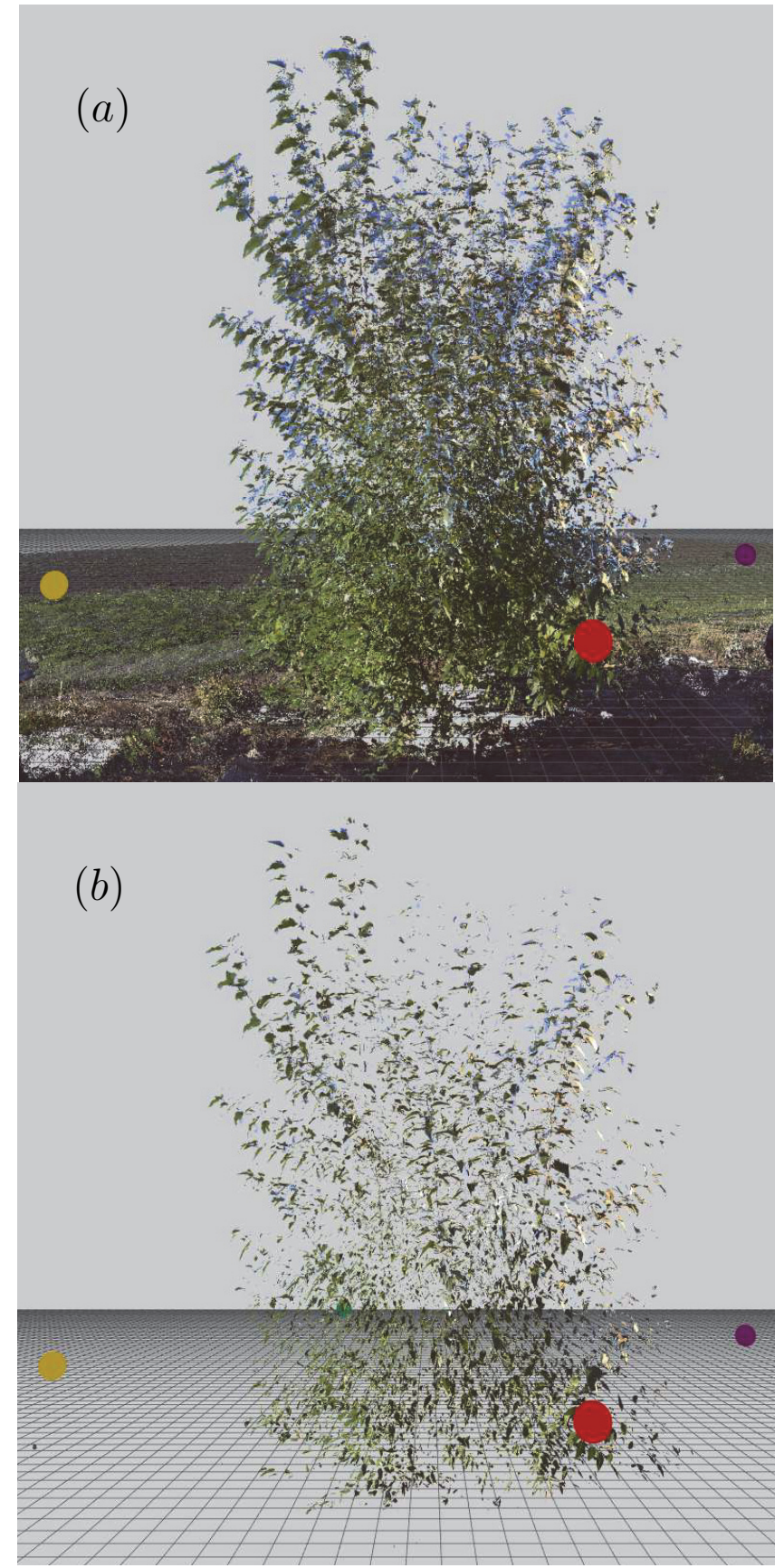

Figure 6: Leaf surface reconstruction of the isolated tree using LiDAR data (low resolution): (a) raw scan points, (b) triangulated surfaces $\left(L_{\max }=5 \mathrm{~cm},>1.3\right.$ million triangles). Spheres denote each of the four individual scan locations. Note that the surfaces of the triangles are colored by linearly interpolating the RGB values given at the vertices by the scanner. 


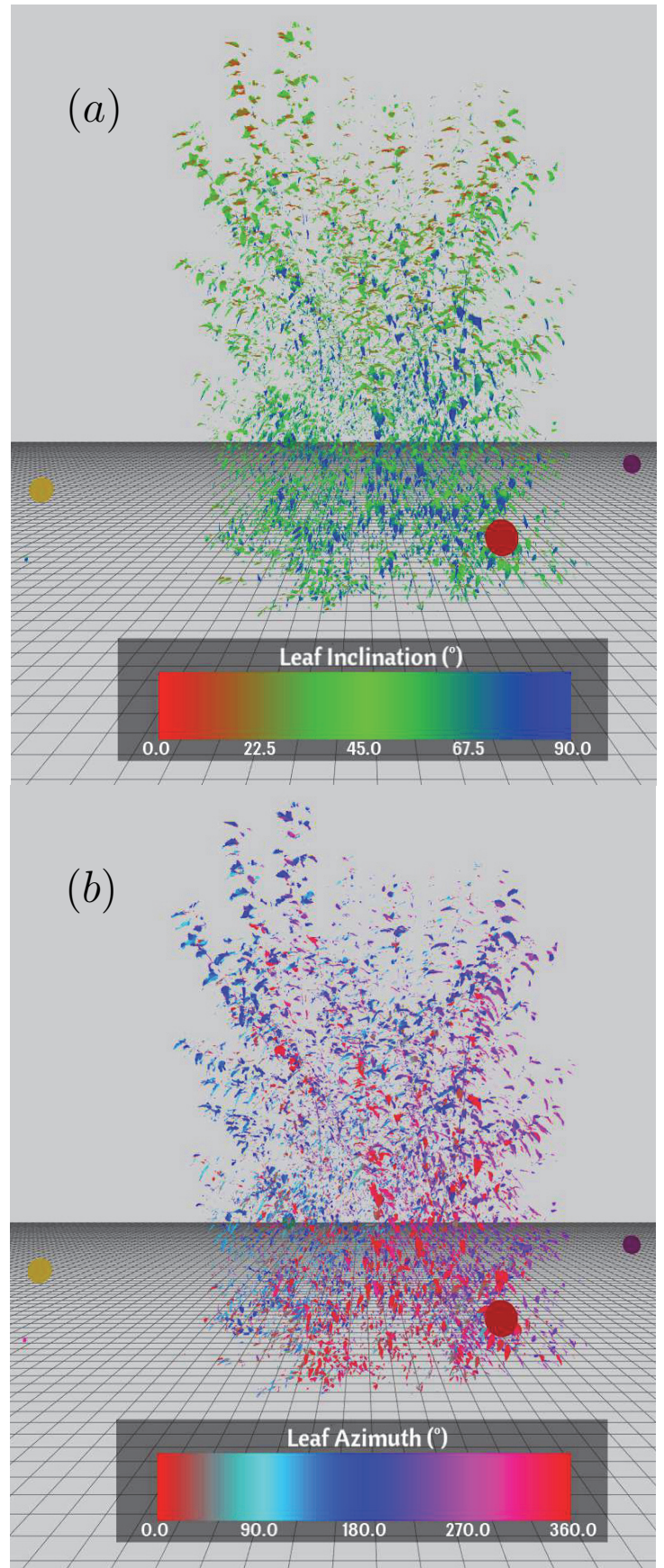

Figure 7: Visualization of the three-dimensional distribution of leaf orientation in the isolated tree. Triangles are colored based on their computed (a) inclination angle, and (b) azimuthal angle. The view shown in the figure is South-facing $\left(180^{\circ}\right)$. Spheres denote each of the four individual scan locations. 

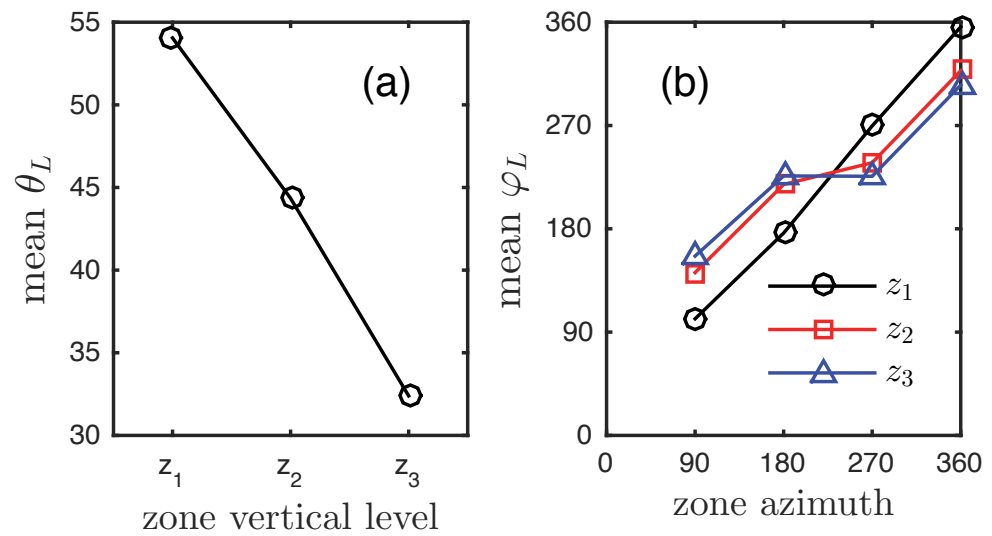

Figure 8: Variation of mean leaf orientation in space. (a) Mean leaf inclination angle for each vertical zone level ( $z_{1}$ closest to ground, $z_{3}$ furthest from ground); (b) Linear directional mean of leaf azimuth for each azimuthal zone at each vertical zone level. 
upper crown (which were less obstructed) had normals that skewed toward the South and were most likely to be inclined at $30-35^{\circ}$ (Fig. 8a), suggesting that they were oriented to maximize sunlight interception. Leaves near the ground were closer to vertical and tended to point along the shortest path length leading to the outer edge of the crown, which would appear to absorb the most sunlight given that dense leaves attenuate light from above.

Overall, good agreement was found between LiDAR and manual measurements of leaf angle distribution functions (Figs. 9 and 10), considering expected errors present in manual measurements. The manually measured PDFs contain noticeable noise, indicating that more than 50 samples per zone are likely needed for a smooth distribution. However, data is of high enough quality to show a clear trend in the PDFs, which qualitatively agrees with the PDFs obtained using the LiDAR measurement technique.

\subsection{Vineyard}

Visualizations of leaf orientation for the vineyard also show an intuitive trend (Fig. 11). The majority of the leaves were inclined at greater than $45^{\circ}$ and had azimuthal angles in approximately the row-normal directions. Calculated PDFs of leaf inclination verified these qualitative observations (Fig. 12), revealing an inclination distribution that is approximately erectophile and an azimuthal distribution that biased toward the row-normal directions.

Like in the isolated tree, LiDAR measurements of leaf angle distribution functions in the vineyard appeared to be skewed toward the direction that will provide the most sunlight interception. The design of the trellis system is intended to maximize light penetration, which directly exposes leaves to sunlight from the sides. Thus, one may intuitively expect leaf orientations to bias toward the row-normal directions and be inclined closer to vertical. It should be reiterated that the tops of the "upper" zones were not located at the top of 

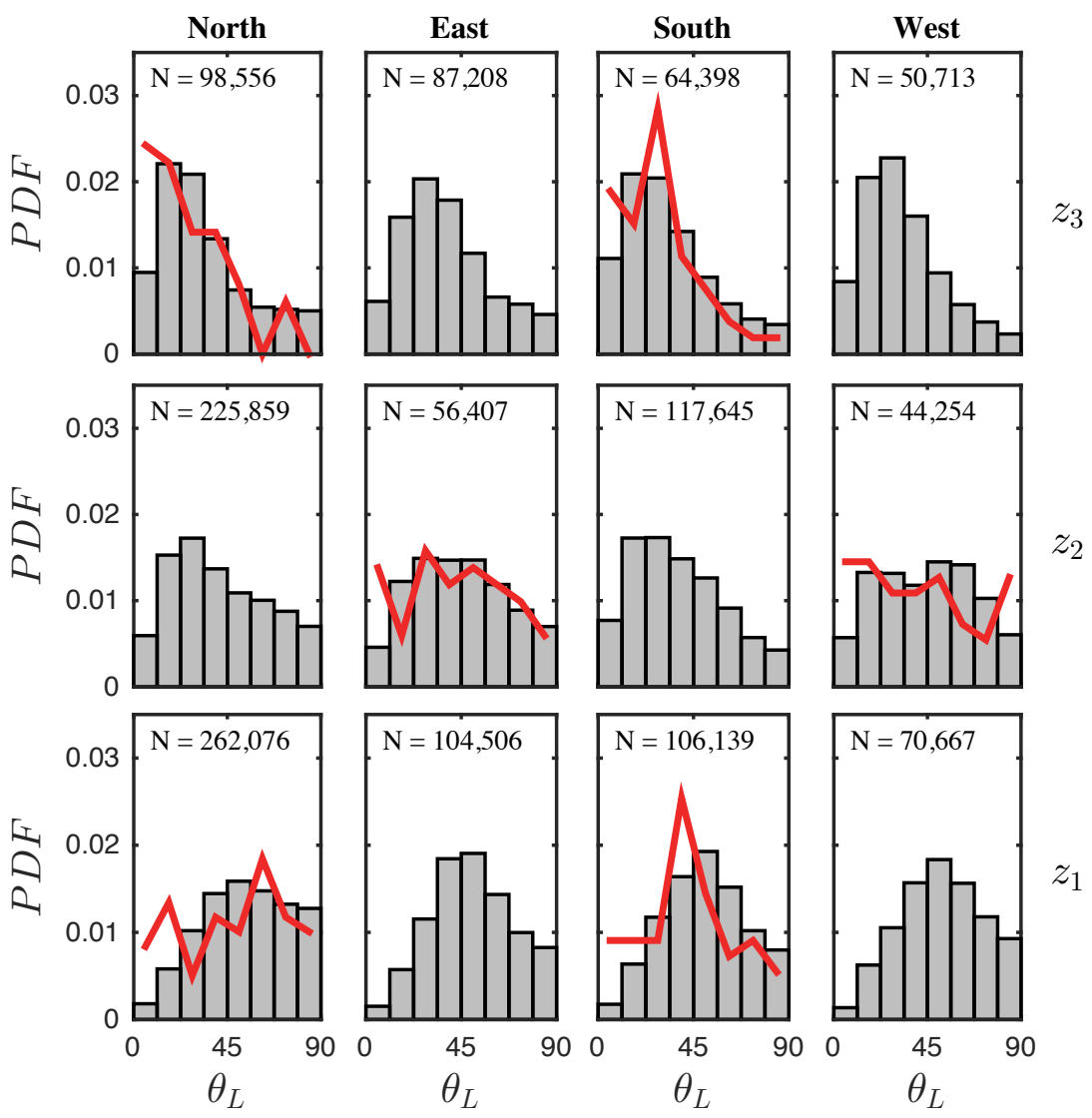

Figure 9: Probability density functions of leaf inclination angle for 12 volumes within an isolated tree. Note that $\theta_{L}=0$ and $\theta_{L}=90^{\circ}$ correspond to horizontal and vertical leaves, respectively. Solid lines represent the manually measured probability density functions (50 samples per zone). Columns correspond to the volume's azimuthal direction (e.g., North is on the North side of the tree). Rows correspond to the volume's vertical level ( $z_{1}$ is lowest, $z_{3}$ is highest). $\mathrm{N}$ denotes the number of samples (i.e., triangles) that comprise the density function. 

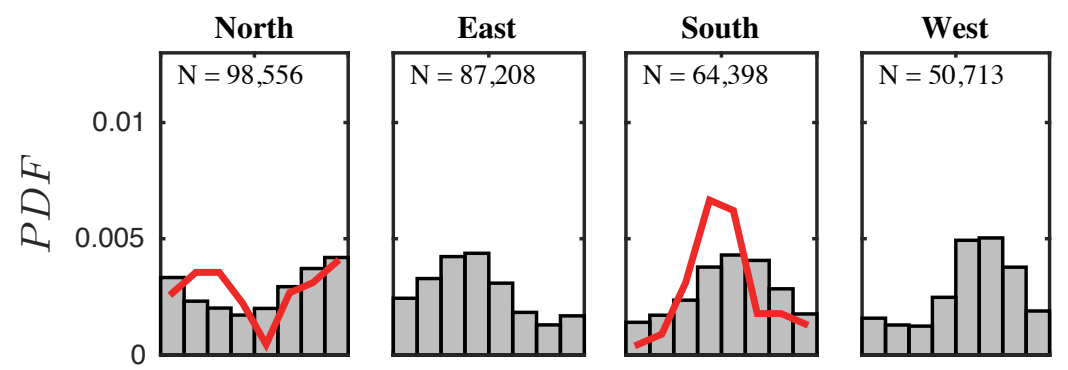

$z_{3}$
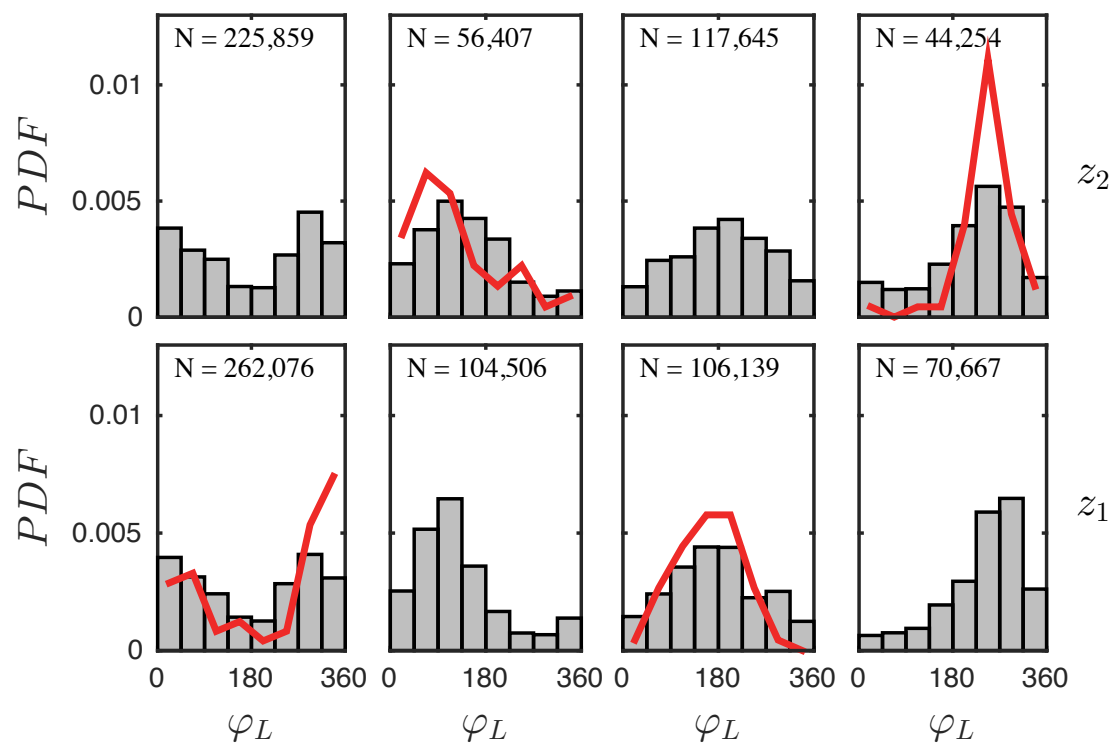

Figure 10: Probability density functions of leaf azimuthal angle for 12 volumes within an isolated tree. Note that $\varphi_{L}=0$ is North, $\varphi=90^{\circ}$ is East, etc. Solid lines represent the manually measured probability density functions (50 samples per zone). Columns correspond to the volume's azimuthal direction (e.g., North is on the North side of the tree). Rows correspond to the volume's vertical level ( $z_{1}$ is lowest, $z_{3}$ is highest). $\mathrm{N}$ denotes the number of samples (i.e., triangles) that comprise the density function. 


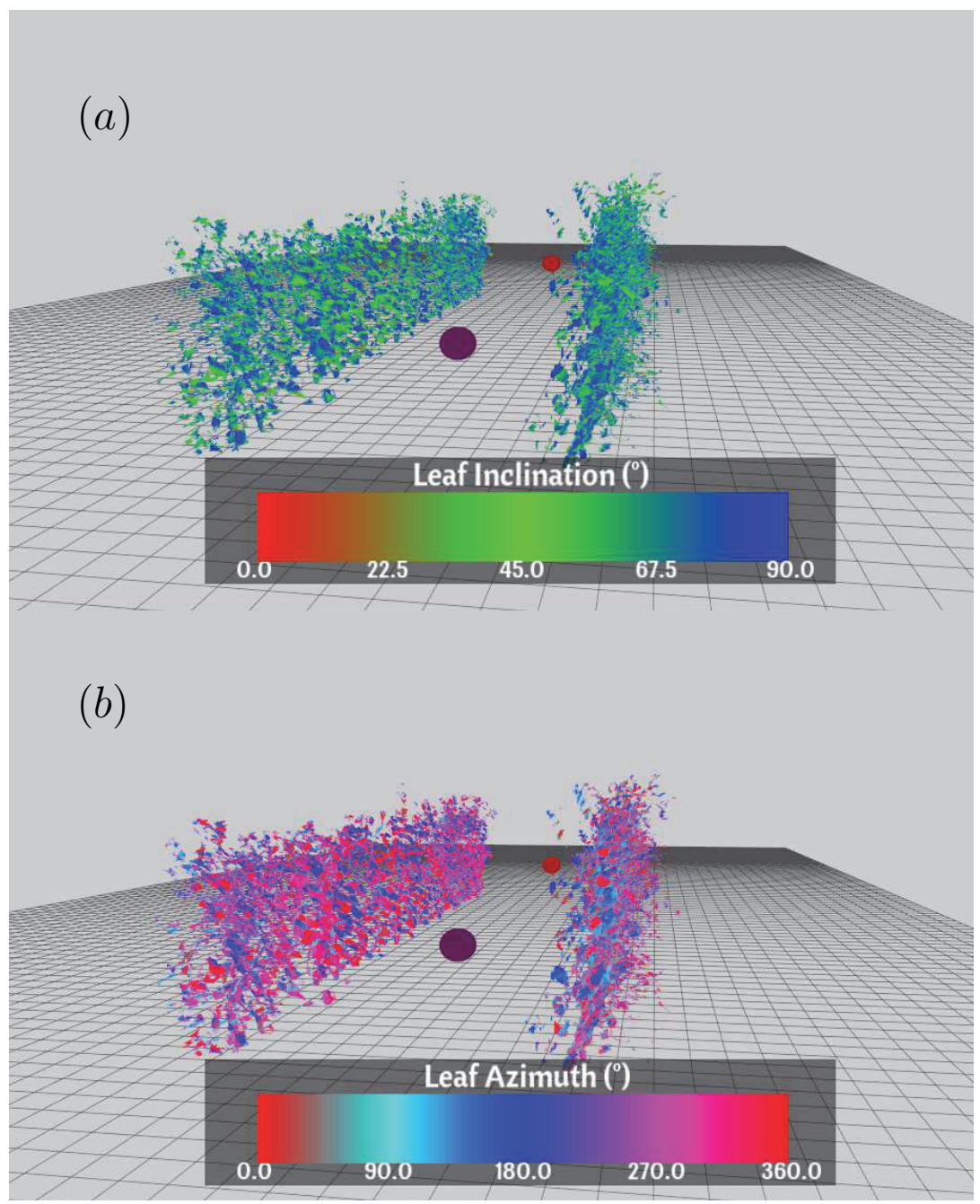

Figure 11: Visualization of the three-dimensional distribution of leaf orientation in the vineyard $\left(L_{\max }=5 \mathrm{~cm}\right)$. Triangles are colored based on their computed (a) inclination angle, and (b) azimuthal angle. The view shown in the figure is approximately South-facing $\left(180^{\circ}\right)$. Spheres denote each of the two individual scan locations. 
vegetation, but rather there was still a significant amount of vegetation above the upper zones. As a result, there was little difference in the PDFs of leaf inclination between the upper and lower zones. The distribution of azimuthal angles is slightly more uniform for the upper zones, which is consistent with results for the isolated tree.

Agreement between LiDAR and manual measurements of leaf orientation was poor. As discussed previously, the vineyard presented some substantial challenges when performing manual measurements of leaf orientation, which was primarily due to complex leaf geometry/topography. This generally resulted in manual measurements that biased toward lower inclination angles. Indeed, the manual measurements of leaf inclination gave a more Gaussian PDF, with the peak lying between $40-60^{\circ}$. It is probable that the manually measured PDF contains substantial errors, and that it is unable to properly represent leaves oriented near vertical. If a qualitative comparison is performed with more rigorous data in a vineyard (e.g., Bailey et al., 2014), it appears that the LiDAR measurements agree with the expected behavior that the PDF of $\theta_{L}$ peaks at around $90^{\circ}$. The PDF of $\theta_{L}$ reported in Bailey et al. (2014) consists of much more detailed measurements that were extremely time consuming to collect. These measurements gave a PDF of $\theta_{L}$ that exponentially decreased away from vertical, which, along with physical intuition and the visualizations in Fig. 11a, agrees with the LiDAR measurements.

The LiDAR and manual measurements of leaf azimuth showed similar trends, but quantitative agreement was not particularly good. Both the manual and LiDAR measurements show peaks in the PDFs in the row-normal directions. However the peaks in the manually measured PDFs are much more pronounced. This can also be explained by the curvature of the leaves. Although the average leaf orientation is most likely to be in the row-normal directions, any curvature 

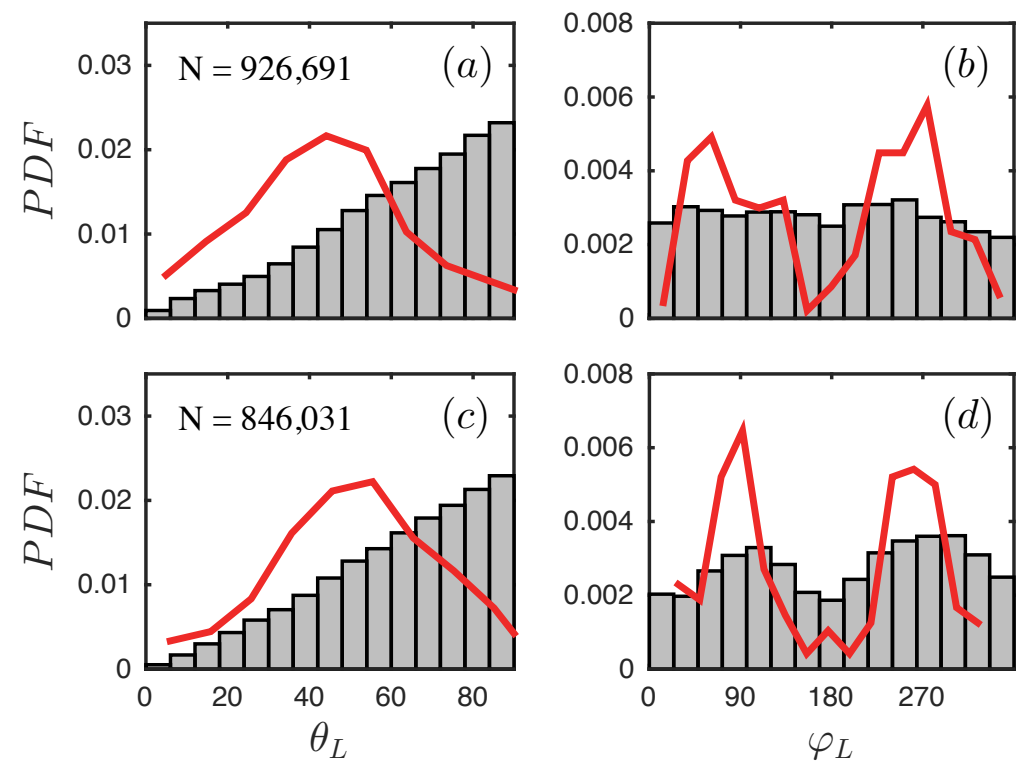

Figure 12: Probability density functions of leaf orientation within a vineyard. Note that the vineyard rows run North-South $\left(\varphi=0,180^{\circ}\right)$. Solid lines represent the manually measured probability density functions (200 samples per zone). (a),(b) correspond to the upper zones, and (c),(d) correspond to the lower zones. $\mathrm{N}$ is the total number of samples used to construct the probability density function (i.e., the number of triangles in either the upper or lower volumes).

in the leaves will give contributions to the off-normal directions. This means that the prominence of the peaks is likely to be reduced if the contributions of all parts of the leaves are considered.

\subsection{Effect of scan resolution}

The effect of the scan resolution was examined by comparing the low, medium, and high density scans. The PDFs obtained from the low resolution LiDAR scans previously shown in Figs. 9 and 10 are compared against the PDFs formed from the mid and high resolution scans in Figs. 13 and 14. It should also be noted that the same length threshold value of $L_{\max }=5 \mathrm{~cm}$ was used for all three resolutions. Overall, changes to the PDFs were minimal as scan resolution was varied, indicating that the technique is relatively robust for a range of scan 
densities. Visual inspection showed that for the mid and high resolution scans, there was some noise in calculated normals due to errors in measured hit point locations, although this did not seem to affect the PDFs. Similar observations were found for the vineyard, although not shown for brevity.

\section{Summary}

This work presents and validates a method that can rapidly measure the three-dimensional distribution of leaf orientation using terrestrial LiDAR scanning data. The LiDAR instrument is placed near the plant to be measured and used to generate a cloud of millions of discrete points that give the distances to various objects surrounding it.

A probability density function (PDF) of leaf orientation can then be computed for any arbitrary volume of vegetation using the triangle normals. It is critical that each normal be correctly weighted in terms of its contribution to the PDF, otherwise the PDF will bias toward normals that are parallel to the ray directions. The PDF should be formed by summing the triangle areas corresponding to discrete bins of $\delta \theta$ or $\delta \varphi$ and normalizing such that the density function integrates to unity.

Primary assumptions and limitations of the technique are:

1. It was assumed that the scan density is such that the characteristic triangle dimension is much smaller than the characteristic leaf dimension and leaf separation dimension, but much larger than the error in the LiDAR distance measurement. The scan density and distance from vegetation should be adjusted to meet this requirement. Alternatively, one could scan at the highest possible density and systematically discard points from the scan such that this requirement is met. 

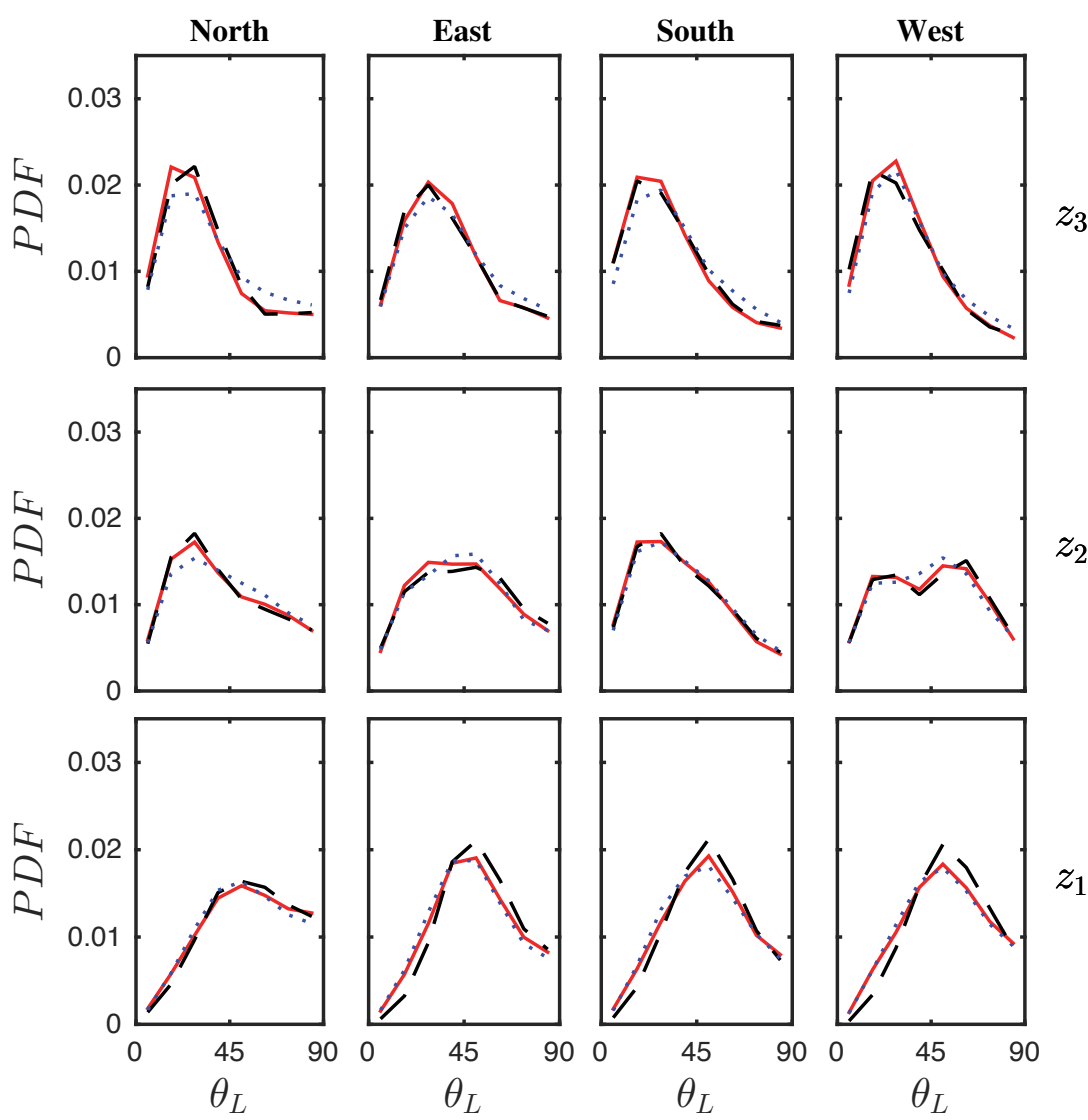

Figure 13: Probability density functions of leaf inclination angle for 12 volumes within an isolated tree for varying scan resolutions: 'low' (solid lines), 'mid' (dashed lines), and 'high' (dotted lines). Note that $\theta_{L}=0$ and $\theta_{L}=90^{\circ}$ correspond to horizontal and vertical leaves, respectively. Columns correspond to the volume's azimuthal direction (e.g., North is on the North side of the tree). Rows correspond to the volume's vertical level (e.g., $z_{1}$ is lowest, $z_{3}$ is highest). 

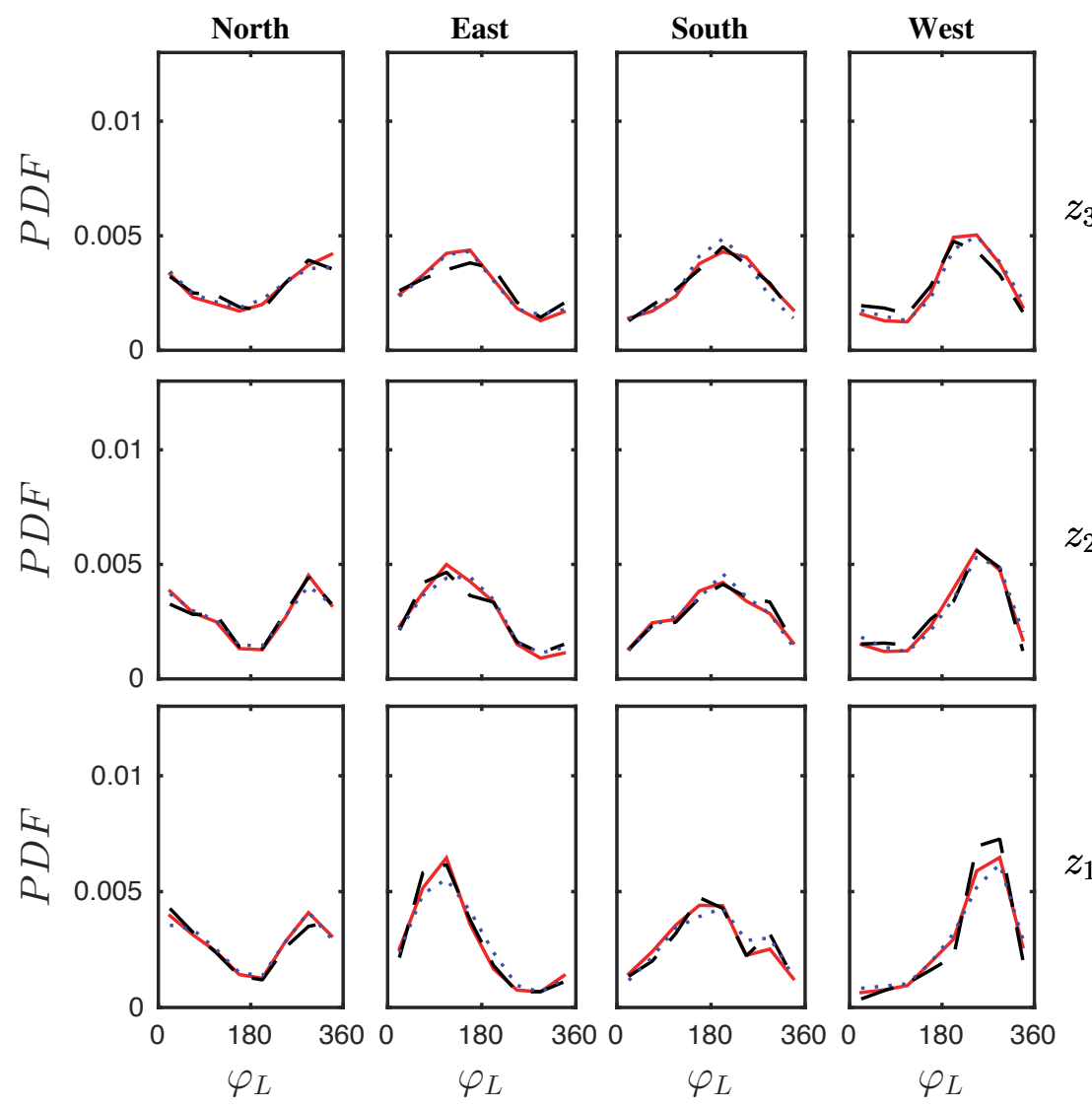

Figure 14: Probability density functions of leaf azimuthal angle for 12 volumes within an isolated tree for varying scan resolutions: 'low' (solid lines), 'mid' (dashed lines), and 'high' (dotted lines). Note that $\varphi_{L}=0$ is North, $\varphi=90^{\circ}$ is East, etc. Columns correspond to the volume's azimuthal direction (e.g., North is on the North side of the tree). Rows correspond to the volume's vertical level (e.g., $z_{1}$ is lowest, $z_{3}$ is highest). 
2. The triangulation algorithm was assumed to adequately remove the effects of partial hits by choosing an $L_{\max }$ value that is sufficiently small. Points could also be filtered using the reflectance of the hits or using an edge detection algorithm.

3. It was assumed that the woody area is small compared to leaf area, or that a distinction between the two in not necessary. If desired, one could use an algorithm to separate hit points corresponding to wood and leaf material (e.g., Béland et al., 2014).

4. Leaves that are completely obstructed from the view of the laser are not measured. Thus, measured leaf orientations are likely to bias toward leaves that are not occluded from the view of the scanner. This can be overcome to some degree by using multiple scanner locations, however it is an inherent limitation of LiDAR (and other optical) measurements in general.

5. Scans should be performed in minimal wind conditions to reduce noise in distance measurements.

Because of the considerable error in field measurements of leaf orientation, the method was demonstrated to be consistent using synthetic scan simulations. These simulations demonstrated the consistency of the method overall, and were used to quantify errors in an ideal situation. It was found that errors in the estimated leaf angle PDF decreased with increasing scan density and as leaf density increased. The methodology was validated in the field by comparing LiDAR-generated measurements to manual measurements in an isolated tree and in a grapevine canopy. Qualitative agreement between manual and LiDAR measurements for the isolated tree was good, and demonstrated the feasibility of the LiDAR technique. Agreement between manual and LiDAR measurements in the grapevine canopy was relatively poor, which was attributed to the qual- 
ity of the manual measurements. Replicant measurements demonstrated large biases in manual measurements when leaf shape/topography was complex. However, behavior of the LiDAR measurements in the vineyard intuitively agreed with visual observations as well as previously published data. It is likely that the LiDAR measurements are at least as accurate, if not more accurate than manual measurements, yet the LiDAR measurements give much more detailed information in a fraction of the time.

Future work should continue to evaluate the limitations and trade-offs of the LiDAR measurement method. The future applications of the method in advancing our understanding of plant physics are vast. Numerous modeling and analytical techniques could be improved by incorporating detailed information related to leaf orientation, ranging from applications in remote sensing to turbulent flow physics. The technique can be used to improve LiDAR measurements of leaf area density distributions by providing the correct projected leaf area fractions. There is also opportunity to analyze the temporal behavior (e.g., heliotropic motion) of leaf orientation by performing many LiDAR scans separated in time.

\section{Acknowledgments}

The authors are indebted to the numerous individuals that assisted in collecting the field data presented in this work: Jesse Mitchell, Tara Neill, Caroline Press, Brenda Shaffer, Lindsey Thiessen, Matt Trappe, Bailey Ward, and Brent Warneke. This research was financially supported by United States Department of Agriculture (USDA) project 5358-22000-039-00D. LiDAR equipment was provided by a grant from the Erath Foundation. The use, trade, firm, or corporation names in this publication are for information and convenience of the reader. Such use does not constitute an endorsement or approval by the USDA 
or the Agricultural Research Service of any product or service to the exclusion of others that may be suitable.

\section{References}

Bailey, B.N., Overby, M., Willemsen, P., Pardyjak, E.R., Mahaffee, W.F., Stoll, R., 2014. A scalable plant-resolving radiative transfer model based on optimized GPU ray tracing. Agric. For. Meteorol. 198-199, 192-208.

Bailey, B.N., Stoll, R., Pardyjak, E.R., Miller, N.E., 2016. A new threedimensional energy balance model for complex plant canopy geometries: Model development and improved validation strategies. Agric. For. Meteorol. 218-219, 146-160.

Béland, M., Baldocchi, D.D., Widlowski, J.L., Fournier, R.A., Verstraete, M.M., 2014. On seeing the wood from the leaves and the role of voxel size in determining leaf area distribution of forests with terrestrial lidar. Agric. For. Meteorol. 184, 82-97.

Béland, M., Widlowski, J.L., Fournier, R.A., Côté, J.F., Verstraete, M.M., 2011. Estimating leaf area distribution in savanna trees from terrestrial LiDAR. Agric. For. Meteorol. 151, 1252-1266.

Bernardini, F., Mittleman, J., Rushmeier, H., Silva, C., Taubin, G., 1999. The ball-pivoting algorithm for surface reconstruction. IEEE Trans. Vis. Comp. Graph. 5, 349-359.

de Wit, C.T., 1965. Photosynthesis of Leaf Canopies. Agricultural Research Report 663. Wageningen. 57 pp.

Henning, J.G., Radtke, P.J., 2006. Ground-based laser imaging for assessing three-dimensional forest canopy structure. Photogramm. Eng. Remote Sens. 72, 1349-1358. 
Hosoi, F., Omasa, K., 2006. Voxel-based 3-D modeling of individual trees for estimating leaf area density using high-resolution portable scanning lidar. IEEE Trans. Geosci. Remote Sens. 40, 3610-3618.

Hosoi, F., Omasa, K., 2015. Estimating leaf inclination distribution of broadleaved trees in each part of the canopies by a high-resolution portable scanning lidar. J. Agric. Meteorol. 71, 136-141.

Jones, H.G., Vaughan, R.A., 2010. Remote Sensing of Vegetation: Principles, Techniques, and Applications. Oxford University Press, Oxford, UK. 400 pp.

Kull, O., Broadmeadow, M., Kruijt, B., Meir, P., 1999. Light distribution and foliage structure in an oak canopy. Trees 14, 55-64.

Lave, M., Kleissl, J., 2011. Optimum fixed orientations and benefits of tracking for capturing solar radiation in the continental United States. Renew. Energy $36,1145-1152$.

Myneni, R.B., Ross, J., 1991. Photon-Vegetation Interactions: Applications in Remote Sensing and Plant Ecology. Springer. 582 pp.

Nilson, T., Kuusk, A., 1989. A reflectance model for the homogeneous plant canopy and its inversion. Remote Sens. Environ. 27, 157-167.

Norman, J.M., Campbell, G.S., 1989. Canopy structure, in: Pearcy, R.W., Ehleringer, J., Mooney, H.A., Rundel, P.W. (Eds.), Plant Physiological Ecology: Field Methods and Instrumentation. Chapman \& Hall, New York, pp. $301-326$.

Pisek, J., Sonnentag, O., Richardson, A.D., ottus, M.M., 2013. Is the spherical leaf inclination angle distribution a valid assumption for temperate and boreal broadleaf tree species? Agric. For. Meteorol. 169, 186-194. 
Press, W.H., Teukolsky, S.A., Vetterling, W.T., Flannery, B.P., 2007. Numerical Recipes: The Art of Scientific Computing. Cambridge University Press, Cambridge, U.K. 1256 pp.

Raabe, K., Pisek, J., Sonnentag, O., Annuk, K., 2015. Variations of leaf inclination angle distribution with height over the growing season and light exposure for eight broadleaf tree species. Agric. For. Meteorol. 214-215, 2-11.

Radtke, P.J., Bolstad, P.V., 2001. Laser point-quadrat sampling for estimating foliage-height profiles in broad-leaved forests. Can. J. For. Res. 31, 410-418.

Raupach, M.R., Woods, N., Dorr, G., Leys, J.F., Cleugh, H.A., 2001. The entrapment of particles by windbreaks. Atmos. Env. 35, 3373-3383.

Rosell, J.R., Llorens, J., Sanz, R., Arnó, J., R.-D., M., Masip, J., Escolà, A., Camp, F., Solanelles, F., Gràcia, F., Gil, E., Val, L., Planas, S., Palacín, J., 2009. Obtaining the three-dimensional structure of tree orchards from remote 2D terrestrial LIDAR scanning. Agric. For. Meteorol. 149, 1505-1515.

Ross, J., 1981. The Radiation Regime and Architecture of Plant Stands. Dr. W. Junk Publishers, The Hague, The Netherlands. 424 pp.

Ryu, Y., Sonnentag, O., Nilson, T., Vargas, R., Kobayashi, H., Wenk, R., Baldocchi, D.D., 2010. How to quantify tree leaf area index in an open savanna ecosystem: A multi-instrument and multi-model approach. Agric. For. Meteorol. 150, 63-76.

Schuepp, P.H., 1993. Tansley review no. 59. leaf boundary layers. New Phytologist $125,477-507$.

Shell, G.S.G., Lang, A.R.G., Sale, P.J.M., 1974. Quantitative measures of leaf orientation and heliotropic response in sunflower, bean, pepper and cucumber. Agric. Meteorol. 13, 25-37. 
Suffern, K.G., 2007. Ray Tracing from the Ground Up. A K Peters/CRC Press, Boca Raton, FL. 784 pp.

Thanisawanyangkura, S., Sinoquet, H., Rivet, P., Cretenet, M., Jallas, E., 1997. Leaf orientation and sunlit leaf area distribution in cotton. Agric. For. Meteorol. $86,1-15$.

Yang, X., Strahler, A.H., Schaaf, C.B., Jupp, D.L.B., Yao, T., Zhao, F., Wang, Z., Culvenor, D.S., Newnham, G.J., Lovell, J.L., Dubayah, R.O., Woodcock, C.E., Ni-Meister, W., 2013. Three-dimensional forest reconstruction and structural parameter retrievals using a terrestrial full-waveform lidar instrument (Echidna@). Remote Sens. Env. 135, 36-51.

Zheng, G., Moskal, L.M., 2012. Leaf orientation retrieval from terrestrial laser scanning (TLS) data. IEEE Trans. Geosci. Remote Sens. 50, 3970-3979.

\section{List of Figures}

1 Visual depiction of point cloud triangulation. (a) shows how points are first triangulated using the two-dimensional grid of $\left(\theta_{i}, \varphi_{j}\right)$ pairs. Leaf-laser intersection points (hits) and non-intersection points (misses) are denoted respectively by closed and open circles. At any hit point, a triangulation is attempted with the $\left(\theta_{i}, \varphi_{j}\right),\left(\theta_{i+1}, \varphi_{j}\right),\left(\theta_{i+1}, \varphi_{j+1}\right)$ points and the $\left(\theta_{i}, \varphi_{j}\right),\left(\theta_{i+1}, \varphi_{j+1}\right)$, $\left(\theta_{i}, \varphi_{j+1}\right)$ points. (b) shows the triangulation mapped to the

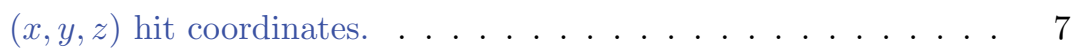

2 Schematic of the coordinate system for two adjacent triangles. . . 9 
3 Assessment of error in manual measurements of leaf orientation in the vineyard canopy. Differing symbols correspond to each of the nine raters, who measured the same 20 leaves in the vineyard. Measurements are reported of the person's view of the average or representative (a) leaf inclination and (b) leaf azimuth. Solid horizontal lines denote the mean observation, and dashed horizontal lines denote \pm 1 standard deviation. Note that some azimuthal measurements in (b) were shifted by $360^{\circ}$ such that they could be visually grouped with other measurements. . . . . . . . . . . . 19

4 Illustration of triangulation methodology using synthetic LiDAR scan data. A visual depiction of the simulated scan with 64 circular disks is shown in (a). Rays are launched from the sphere, some of which intersect the disks, which are visualized using the triangulation. Probability density functions are calculated when no weighting is applied and when a proper area weighting is applied, which are compared to the 'exact' distributions. (b,c) 2 disks, and $(\mathrm{d}, \mathrm{e}) 27$ disks. . . . . . . . . . . . . . . 23

5 Box-and-whisker plots of normalized error in the PDF of $(\mathrm{a}, \mathrm{c}) \theta_{L}$ and (b,d) $\varphi_{L}$ for the synthetic scan cases. The normalized error is defined as the root-mean-squared error between the exact PDF and the PDF formed by the triangulation methodology, which is normalized by the probability density for a uniform distribution (i.e., $1 / 90$ for $\theta_{L}$ and $1 / 360$ for $\varphi_{L}$ ). . . . . . . . . . . 
Leaf surface reconstruction of the isolated tree using LiDAR data (low resolution): (a) raw scan points, (b) triangulated surfaces ( $L_{\max }=5 \mathrm{~cm},>1.3$ million triangles). Spheres denote each of the four individual scan locations. Note that the surfaces of the triangles are colored by linearly interpolating the RGB values given at the vertices by the scanner. . . . . . . . . . .

7 Visualization of the three-dimensional distribution of leaf orientation in the isolated tree. Triangles are colored based on their computed (a) inclination angle, and (b) azimuthal angle. The view shown in the figure is South-facing $\left(180^{\circ}\right)$. Spheres denote each of the four individual scan locations. . . . . . . . . . 26

8 Variation of mean leaf orientation in space. (a) Mean leaf inclination angle for each vertical zone level $\left(z_{1}\right.$ closest to ground, $z_{3}$ furthest from ground); (b) Linear directional mean of leaf azimuth for each azimuthal zone at each vertical zone level. . . . . 27

9 Probability density functions of leaf inclination angle for 12 volumes within an isolated tree. Note that $\theta_{L}=0$ and $\theta_{L}=90^{\circ}$ correspond to horizontal and vertical leaves, respectively. Solid lines represent the manually measured probability density functions (50 samples per zone). Columns correspond to the volume's azimuthal direction (e.g., North is on the North side of the tree). Rows correspond to the volume's vertical level $\left(z_{1}\right.$ is lowest, $z_{3}$ is highest). $\mathrm{N}$ denotes the number of samples (i.e., triangles) that comprise the density function. . . . . . . . . . . . . . . . 29 
10 Probability density functions of leaf azimuthal angle for 12 volumes within an isolated tree. Note that $\varphi_{L}=0$ is North, $\varphi=90^{\circ}$ is East, etc. Solid lines represent the manually measured probability density functions (50 samples per zone). Columns correspond to the volume's azimuthal direction (e.g., North is on the North side of the tree). Rows correspond to the volume's vertical level ( $z_{1}$ is lowest, $z_{3}$ is highest). $\mathrm{N}$ denotes the number of samples (i.e., triangles) that comprise the density function. . . .

11 Visualization of the three-dimensional distribution of leaf orientation in the vineyard $\left(L_{\max }=5 \mathrm{~cm}\right)$. Triangles are colored based on their computed (a) inclination angle, and (b) azimuthal angle. The view shown in the figure is approximately South-facing $\left(180^{\circ}\right)$. Spheres denote each of the two individual scan locations.

12 Probability density functions of leaf orientation within a vineyard. Note that the vineyard rows run North-South $\left(\varphi=0,180^{\circ}\right)$. Solid lines represent the manually measured probability density functions (200 samples per zone). (a),(b) correspond to the upper zones, and (c),(d) correspond to the lower zones. $\mathrm{N}$ is the total number of samples used to construct the probability density function (i.e., the number of triangles in either the upper or lower volumes). . . . . . . . . . . . . . . . 
13 Probability density functions of leaf inclination angle for 12 volumes within an isolated tree for varying scan resolutions: 'low' (solid lines), 'mid' (dashed lines), and 'high' (dotted lines). Note that $\theta_{L}=0$ and $\theta_{L}=90^{\circ}$ correspond to horizontal and vertical leaves, respectively. Columns correspond to the volume's azimuthal direction (e.g., North is on the North side of the tree). Rows correspond to the volume's vertical level (e.g., $z_{1}$ is lowest, $z_{3}$ is highest). . . . . . . . . . . . . . . . .

14 Probability density functions of leaf azimuthal angle for 12 volumes within an isolated tree for varying scan resolutions: 'low' (solid lines), 'mid' (dashed lines), and 'high' (dotted lines). Note that $\varphi_{L}=0$ is North, $\varphi=90^{\circ}$ is East, etc. Columns correspond to the volume's azimuthal direction (e.g., North is on the North side of the tree). Rows correspond to the volume's vertical level (e.g., $z_{1}$ is lowest, $z_{3}$ is highest). . . . . . . . . . . 36 